\title{
SITUAÇÃO PROFISSIONAL DOS LICENCIADOS EM GESTÃO E DESENVOLVIMENTO SOCIAL PELA UCP - PÓLO DE VISEU
}

\begin{abstract}
Pretende apresentar-se, de um modo sucinto, sem utilizar uma análise estatística elaborada, a situação perante o emprego dos licenciados em Gestão e Desenvolvimento Social, pelo Instituto Universitário de Desenvolvimento e Promoção Social (IUDPS) - Universidade Católica Portuguesa (UCP) - Pólo de Viseu, nos anos lectivos de 1995/96, 1996/97 e 1997/98.

$A$ análise incide, essencialmente, sobre a taxa de desemprego, o tempo de espera entre a conclusão da licenciatura e a concretização do emprego, a distribuição do emprego por tipo de actividade e diferenças relevantes entre os licenciados nos vários anos lectivos. $A$ avaliação destes parâmetros resulta num cômputo geral bastante positivo para os licenciados em Gestão e Desenvolvimento Social.
\end{abstract}

Paulo Almeida Pereira*

\section{INTRODUÇÃO AO PROCESSO DE AMOSTRAGEM}

A População ou Universo (conceito estatístico que pode ser definido por um conjunto de unidades com características comuns) que está em análise é constituída por todos os licenciados em Gestão e Desenvolvimento Social $(G D S)$, que obtiveram aquele grau no decorrer dos anos lectivos de 1995/96, 1996/97 e 1997/98 (desde 1 de Outubro de 1995 até ao final do ano de 1998). Cada licenciado constitui uma unidade básica da população: um elemento da população.

* Instituto Universitário de Desenvolvimento e Promoção Social - Pólo de Viseu do Centro Regional das Beiras da Universidade Católica Portuguesa. 
A licenciatura em GDS passou, ao longo do seu percurso de 15 anos por algumas reestruturações, nomeadamente nos anos lectivos de 1992/93 e 1994/95, de modo a responder à evolução do conhecimento científico e pedagógico na área e às solicitações do meio envolvente. A população sobre a qual incide este estudo foi constituída pelos licenciados a partir do ano lectivo de 1995/96 com o objectivo de fazer incidir esta análise sobre os licenciados após a reestruturação da licenciatura.

Há interesse em obter informação sobre características da população em estudo, representadas por variáveis aleatórias que, para um elemento da população, apresentam uma distribuição de probabilidade. No estudo destas variáveis aleatórias e distribuições, a partir de resultados de uma amostra, procura chegar-se a um modelo probabilístico.

Esta recolha de informação pode ser realizada por amostragem. Para obter uma amostra (subconjunto representativo de uma população) existem vários métodos descritos na literatura (Vicente et al., 1996). A partir dos resultados de uma amostra procura chegar-se a um modelo probabilístico que traduza as características em análise da população estudada.

A elaboração do processo de amostragem passou pelos seguintes passos:

1 - Definição dos objectivos do estudo, para a População de licenciados em $G D S$ em análise, que incluem a avaliação de vários tópicos considerados importantes:

- taxa de desemprego;

- distribuição do emprego por tipo de actividade exercida;

- tempo de espera entre a conclusão da licenciatura e a concretização do primeiro emprego;

- diferenças existentes para licenciados em anos diferentes.

2 - Definição do instrumento de recolha, que neste caso é um questionário estruturado, aplicado à população em análise, no decorrer do mês de Maio de 1999.

3 - Definição de um processo de amostragem adequado ao instrumento de análise:

- A população alvo coincide com a população inquirida.

- O questionário estruturado é enviado, por correio, a toda a população em análise. É anexado um envelope endereçado para resposta (taxa paga) de modo a maximizar a taxa de respostas. Este método de recolha de informação apresenta normalmente uma considerável taxa de não-respostas e não garante a aleatoriedade da amostra.

A amostra, para este estudo, é constituída pelo conjunto dos elementos da população que responderam ao questionário. A taxa de não-respostas deve-se a dois motivos: 
- o facto de alguns dos questionários terem sido devolvidos, por não constarem no ficheiro de dados, da Universidade Católica Portuguesa, dos licenciados em $G D S$, as residências actualizadas de alguns elementos da população (o que correspondeu a apenas $5 \%$ da população);

- na sua grande maioria (51\% da população), ao facto de os inquiridos, pura e simplesmente, não responderem.

Por estes factos, a amostra obtida não é uma amostra aleatória, podendo daí resultar algum enviesamento, na análise dos resultados, que não é possível ser quantificado.

Em resumo, os resultados apresentados de seguida foram recolhidos, durante o mês de Maio de 1999, por aplicação de questionários, enviados por correio, ao universo dos alunos licenciados nos anos lectivos de 1995/96, 1996/97, 1997/98 (184 licenciados), tendo sido recebidas 81 respostas, correspondendo a uma amostra de $44 \%$ da população.

\section{APRESENTAÇÃO DOS RESULTADOS}

\subsection{Taxa de desemprego}

No Quadro I apresentam-se, desagregados por ano lectivo em que concluíram a licenciatura, o número de licenciados, o número de inquiridos que responderam ao questionário e os resultados, em frequências absolutas de respostas, relativamente à situação dos licenciados em $G D S$, em termos de emprego. $\mathrm{O}$ número de licenciados ainda desempregados refere-se a quem não teve, até ao momento deste estudo, qualquer emprego e os actualmente desempregados são os que já estiveram empregados.

\section{Quadro I}

LICENCIADOS, NÚMERO DE RESPOSTAS E SITUAÇÃO EM TERMOS DE EMPREGO POR ANO LECTIVO (FREQUÊNCIAS ABSOLUTAS)

\begin{tabular}{cccccc}
\hline Ano de & $\mathbf{N}^{\circ}$ de & $\mathbf{N}^{\circ}$ de & & \multicolumn{2}{c}{ Desempregados } \\
\cline { 5 - 6 } conclusão & licenciados & respostas & Empregados & Actualmente & Ainda \\
\hline $1995 / 96$ & 67 & 21 & 20 & - & 1 \\
$1996 / 97$ & 59 & 22 & 17 & 4 & 1 \\
$1997 / 98$ & 58 & 38 & 33 & 2 & 3 \\
\hline TOTAL & 184 & 81 & 70 & 6 & 5 \\
\hline
\end{tabular}


No Quadro II são transformadas as frequências absolutas apresentadas anteriormente em frequências relativas, com os valores em percentagem. Saliente-se que a percentagem de respostas é reportada ao número total de licenciados, enquanto os restantes valores se reportam ao número de respostas.

\section{Quadro II}

LICENCIADOS, NÚMERO DE RESPOSTAS E SITUAÇÃO

EM TERMOS DE EMPREGO POR ANO LECTIVO (FREQUÊNCIAS RELATIVAS, EM PERCENTAGEM)

\begin{tabular}{ccccc}
\hline Ano de & $\%$ de & & \multicolumn{2}{c}{ Desempregados } \\
\cline { 3 - 5 } conclusão & respostas & Empregados & Actualmente & Ainda \\
\hline $1995 / 96$ & $31 \%$ & $95 \%$ & $0 \%$ & $5 \%$ \\
$1996 / 97$ & $37 \%$ & $77 \%$ & $18 \%$ & $5 \%$ \\
$1997 / 98$ & $66 \%$ & $87 \%$ & $5 \%$ & $8 \%$ \\
\hline TOTAL & $\mathbf{4 4 \%}$ & $\mathbf{8 7 \%}$ & $\mathbf{7 \%}$ & $\mathbf{6 \%}$ \\
\hline
\end{tabular}

Estes resultados são ilustrados pelos Gráficos I, II e II.

\section{Gráfico I}

TAXA DE RESPOSTAS

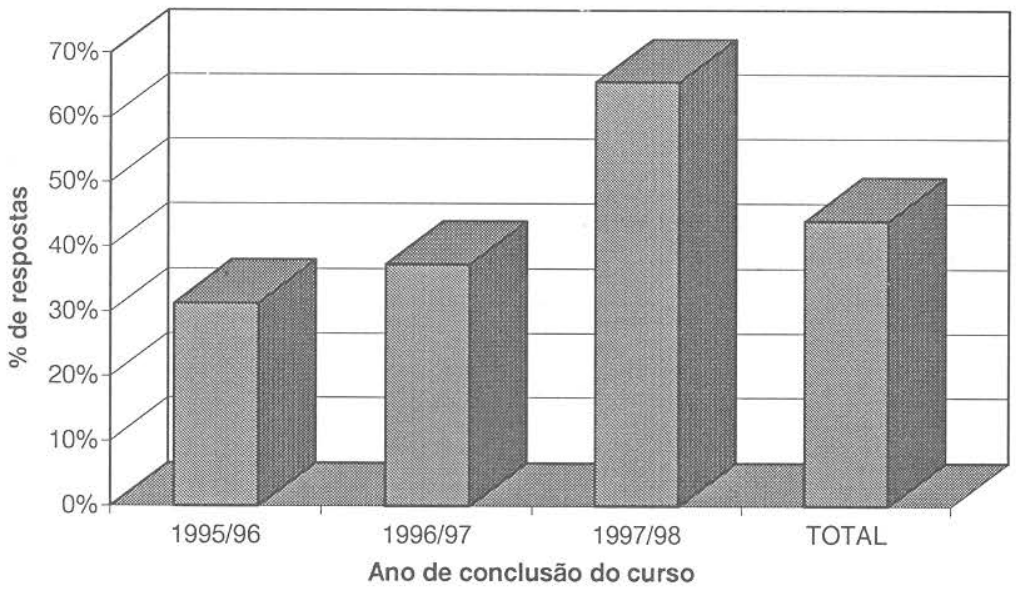




\section{Gráfico II \\ TAXA DE DESEMPREGO}

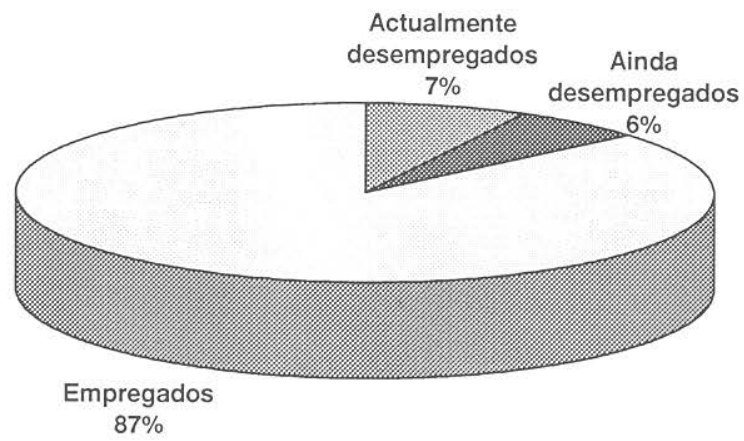

Gráfico III

TAXA DE DESEMPREGO POR ANO LECTIVO

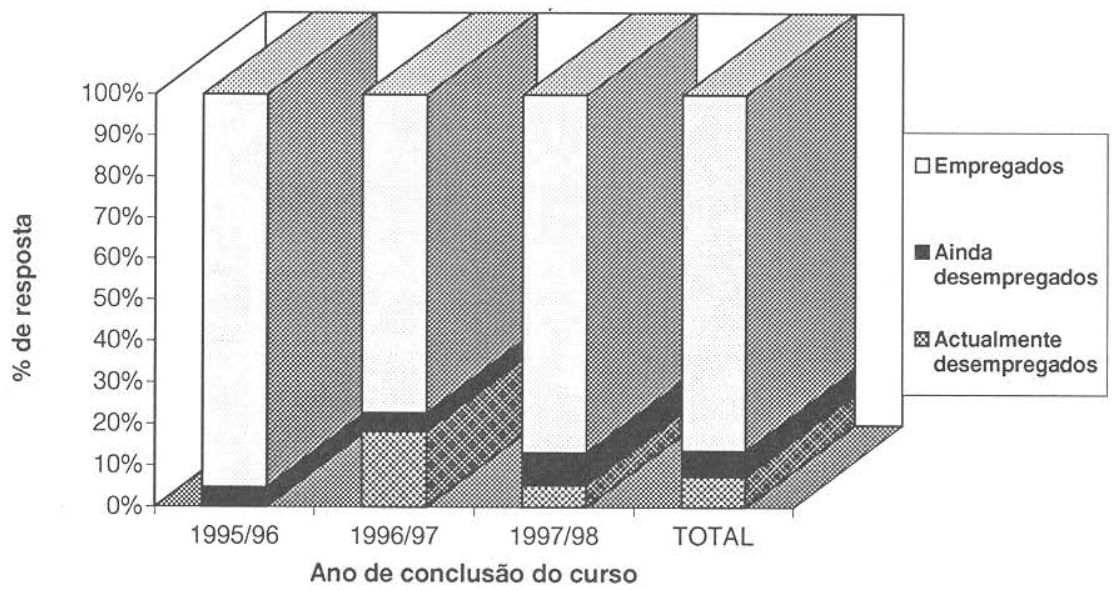

Dos Quadros e Gráficos anteriores, podemos verificar que o número de licenciados diminuiu ligeiramente de 1995/96 para 1996/97, tendo-se mantido constante no ano seguinte. A percentagem de respostas aumenta para os licenciados mais recentes, o que seria de esperar, pois há uma maior 
proximidade com a Universidade. Há que salientar que $87 \%$ do total da amostra se encontra a exercer uma actividade profissional e 7\% já estiveram empregados e estarão presentemente entre empregos. Em termos de desemprego, os licenciados no ano lectivo de 1996/97 são os que apresentam uma maior taxa, embora grande parte deles já tenha exercido uma actividade profissional.

\subsection{Tempo de espera entre a licenciatura e a concretização do primeiro emprego}

Apresentam-se, nos Quadros III e IV, os resultados obtidos para os tempos de espera entre a conclusão da licenciatura e a concretização do primeiro emprego, representados por $t$, em meses. As frequências relativas do Quadro IV dizem respeito apenas aos licenciados que estão empregados.

Note-se que $t<0$ significa que o licenciado já se encontrava a exercer uma actividade profissional no decorrer da licenciatura. Nas colunas seguintes representam-se os licenciados que se empregaram no primeiro mês, no primeiro semestre, no primeiro ano e mais do que um ano após a conclusão da licenciatura.

A última coluna dos Quadros dá-nos uma ideia sobre a rotação no trabalho, através dos licenciados que se mantêm no primeiro emprego que conseguiram.

\section{Quadro III}

TEMPO DE ESPERA ENTRE A LICENCIATURA E O PRIMEIRO EMPREGO (FREQUÊNCIAS ABSOLUTAS)

\begin{tabular}{ccccccc}
\hline Ano de & \multicolumn{7}{c}{$t$ - Tempo entre o fim do curso e o $1^{\circ}$ emprego (meses) } & Ainda no \\
\cline { 2 - 6 } conclusão & $t<0$ & $0<t<1$ & $1<t<6$ & $6<t<12$ & $>12$ & $1^{\circ}$ emprego \\
\hline $1995 / 96$ & 7 & 4 & 3 & 4 & 2 & 10 \\
$1996 / 97$ & 8 & 4 & 5 & - & - & 11 \\
$1997 / 98$ & 14 & 7 & 9 & 1 & 2 & 24 \\
\hline TOTAL & 29 & $\mathbf{1 5}$ & $\mathbf{1 7}$ & $\mathbf{5}$ & $\mathbf{4}$ & $\mathbf{4 5}$ \\
\hline
\end{tabular}




\section{Quadro IV}

TEMPO DE ESPERA ENTRE A LICENCIATURA E O PRIMEIRO EMPREGO (FREQUÊNCIAS RELATIVAS, EM PERCENTAGEM)

\begin{tabular}{ccccccc}
\hline Ano de & \multicolumn{2}{c}{$t$ - Tempo entre o fim do curso e o $1^{\circ}$ emprego (meses) } & Ainda no \\
\cline { 2 - 6 } conclusão & $t<0$ & $0<t<1$ & $1<t<6$ & $6<t<12$ & $t>12$ & $1^{\circ}$ emprego \\
\hline $1995 / 96$ & $35 \%$ & $20 \%$ & $15 \%$ & $20 \%$ & $10 \%$ & $50 \%$ \\
$1996 / 97$ & $47 \%$ & $24 \%$ & $29 \%$ & $0 \%$ & $0 \%$ & $65 \%$ \\
$1997 / 98$ & $42 \%$ & $21 \%$ & $27 \%$ & $3 \%$ & $6 \%$ & $73 \%$ \\
\hline TOTAL & $\mathbf{4 2 \%}$ & $\mathbf{2 1 \%}$ & $\mathbf{2 4 \%}$ & $\mathbf{7 \%}$ & $\mathbf{6 \%}$ & $\mathbf{6 4 \%}$ \\
\hline
\end{tabular}

Alguns dos resultados apresentados são ilustrados pelos Gráficos IV e V.

\section{Gráfico IV}

TEMPO DE ESPERA ENTRE A LICENCIATURA E A CONCRETIZAÇÃO DO PRIMEIRO EMPREGO

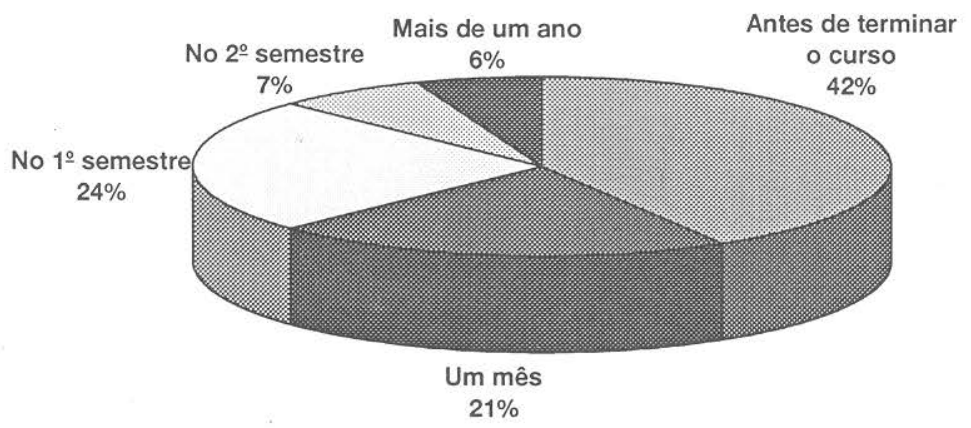




\section{Gráfico V \\ ROTAÇÃO NO TRABALHO: TOTAL E POR ANO LECTIVO}

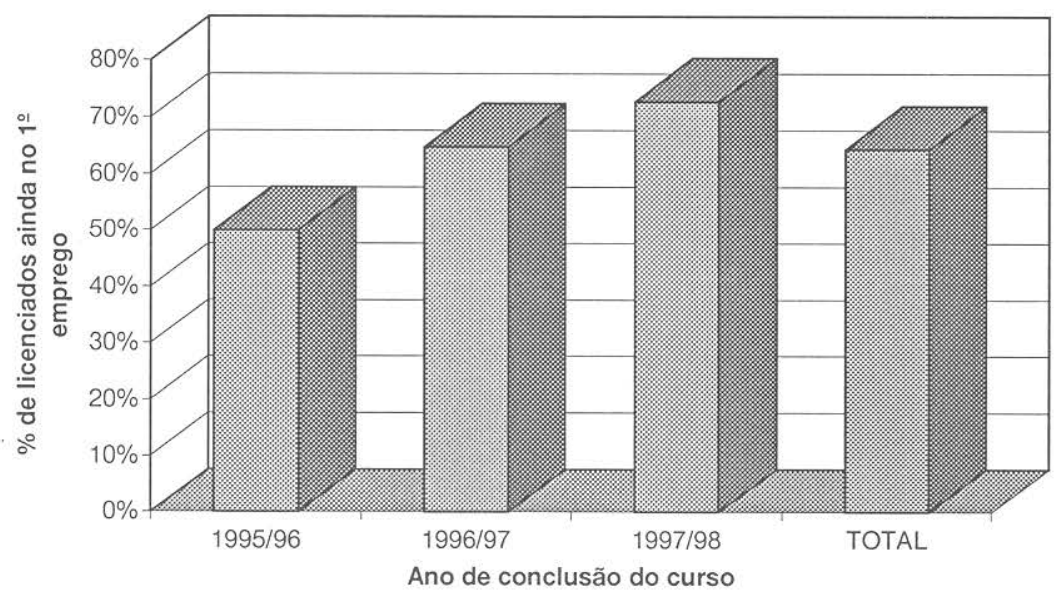

Nos últimos Quadros e Gráficos verifica-se que grande parte dos licenciados (42\%) se encontram empregados ainda antes de concluírem a licenciatura. Uma parte significativa conseguiu o primeiro emprego logo após a conclusão da licenciatura, no espaço de 1 mês ou de 6 meses. Apenas $13 \%$ demoraram mais de um semestre para concretizar o primeiro emprego. Analisando os mesmos resultados, desagregados por ano lectivo, verifica-se que os licenciados no decorrer do ano lectivo 1995/96 são os que apresentam uma percentagem maior de empregados, mais do que um semestre após a conclusão da licenciatura (30\%). Este facto poderá justificar-se, em parte, pelo facto de ter sido maior o número de licenciados neste ano lectivo.

Quanto à rotação no emprego, ela em média é baixa, uma vez que $64 \%$ dos licenciados se mantêm ainda no primeiro emprego. Como seria de esperar a percentagem de licenciados que se mantém ainda naquele aumenta para os licenciados mais recentes.

\subsection{Colocação dos licenciados por tipo de actividade}

Nos Quadros V e VI apresentam-se os resultados obtidos para a colocação dos licenciados em GDS pelos diferentes tipos de actividade em que exercem a sua actividade profissional. 


\section{Quadro V \\ COLOCAÇÃO DOS LICENCIADOS POR TIPO DE ACTIVIDADE (FREQUÊNCIAS ABSOLUTAS)}

\begin{tabular}{ccccccc}
\hline Ano concl. & Ensino & Banca & Seguros & Out.serv. Indústria & Outros \\
\hline $1995 / 96$ & 1 & 2 & 2 & 11 & 4 & \\
$1996 / 97$ & 2 & 4 & 1 & 8 & 1 & 1 \\
$1997 / 98$ & & 7 & & 17 & 9 & \\
\hline TOTAL & $\mathbf{3}$ & $\mathbf{1 3}$ & $\mathbf{3}$ & $\mathbf{3 6}$ & $\mathbf{1 4}$ & $\mathbf{1}$ \\
\hline
\end{tabular}

LEGENDA:

Out.Serv. - outros serviços

\section{ESPECIFICAÇÃO DOS OUTROS SERVIÇOS}

\begin{tabular}{ccccccc}
\hline Ano concl. & A. com. & S./D. & A. empr. & F.públ. & Téc.Sup. & C/A/F \\
\hline $1995 / 96$ & 5 & 1 & 1 & 3 & & \\
$1996 / 97$ & 2 & 1 & & 1 & & 2 \\
$1997 / 98$ & 3 & 1 & & 2 & 1 & 4 \\
\hline TOTAL & $\mathbf{1 0}$ & $\mathbf{3}$ & $\mathbf{1}$ & $\mathbf{6}$ & $\mathbf{1}$ & $\mathbf{6}$ \\
\hline
\end{tabular}

LEGENDA:

A. com. - área comercial

A. empr. - associações empresariais

Téc. Sup. - técnica superior
S./D. - Área social / associações de desenvolvimento

F. públ. - função pública

C/A/F - contabilidade/auditoria/fiscalidade

\section{ESPECIFICAÇÃO DOS OUTROS SERVIÇOS (CONTINUAÇÃO)}

\begin{tabular}{ccccccc}
\hline Ano concl. & Inform. & Admin. & N/T & Inf. méd. Hotelaria & Transp. \\
\hline $1995 / 96$ & - & 1 & - & - & - & - \\
$1996 / 97$ & 1 & 1 & - & - & - & - \\
$1997 / 98$ & - & 1 & 2 & 1 & 1 & 1 \\
\hline TOTAL & $\mathbf{1}$ & $\mathbf{3}$ & $\mathbf{2}$ & $\mathbf{1}$ & $\mathbf{1}$ & $\mathbf{1}$ \\
\hline
\end{tabular}

\section{LEGENDA:}

Inform. - informática

Admin. - administrativos

N/T - notário/tribunal

Inf. méd. - informação médica

Transp. - Transportes 
Quadro VI

COLOCAÇÃO DOS LICENCIADOS POR TIPO DE ACTIVIDADE (FREQUÊNCIAS RELATIVAS, EM PERCENTAGEM)

\begin{tabular}{ccccccc}
\hline Ano concl. & Ensino & Banca & Seguros & Out.serv. & Indústria & Outros \\
\hline $1995 / 96$ & $\mathbf{5 \%}$ & $10 \%$ & $10 \%$ & $55 \%$ & $20 \%$ & $0 \%$ \\
$1996 / 97$ & $12 \%$ & $24 \%$ & $6 \%$ & $47 \%$ & $6 \%$ & $6 \%$ \\
$1997 / 98$ & $0 \%$ & $21 \%$ & $0 \%$ & $52 \%$ & $27 \%$ & $0 \%$ \\
\hline TOTAL & $\mathbf{4 \%}$ & $\mathbf{1 9 \%}$ & $\mathbf{4 \%}$ & $\mathbf{5 1 \%}$ & $\mathbf{2 0 \%}$ & $\mathbf{1 \%}$ \\
\hline
\end{tabular}

LEGENDA:

Out.Serv. - outros serviços

\section{ESPECIFICAÇÃO DOS OUTROS SERVIÇOS}

\begin{tabular}{ccccccc}
\hline Ano concl. & A. com. & S./D. & A. empr. & F.públ. & Téc.Sup. & C/A/F \\
\hline $1995 / 96$ & $25 \%$ & $5 \%$ & $5 \%$ & $15 \%$ & $0 \%$ & $0 \%$ \\
$1996 / 97$ & $12 \%$ & $6 \%$ & $0 \%$ & $6 \%$ & $0 \%$ & $12 \%$ \\
$1997 / 98$ & $9 \%$ & $3 \%$ & $0 \%$ & $6 \%$ & $3 \%$ & $12 \%$ \\
\hline TOTAL & $\mathbf{1 4 \%}$ & $\mathbf{4 \%}$ & $\mathbf{1 \%}$ & $\mathbf{9 \%}$ & $\mathbf{1 \%}$ & $\mathbf{9 \%}$ \\
\hline
\end{tabular}

LEGENDA:

A. com. - área comercial

A. empr. - associações empresariais

Téc. Sup. - técnica superior
S.JD. - Área social / associaçōes de desenvolvimento

F. públ. - função pública

C/A/F - contabilidade/auditoria/fiscalidade

\section{ESPECIFICAÇÃO DOS OUTROS SERVIÇOS (CONTINUAÇÃO)}

\begin{tabular}{ccccccc}
\hline Ano concl. & Inform. & Admin. & N/T & Inf. méd. & Hotelaria & Transp. \\
\hline $1995 / 96$ & $0 \%$ & $5 \%$ & $0 \%$ & $0 \%$ & $0 \%$ & $0 \%$ \\
$1996 / 97$ & $6 \%$ & $6 \%$ & $0 \%$ & $0 \%$ & $0 \%$ & $0 \%$ \\
$1997 / 98$ & $0 \%$ & $3 \%$ & $6 \%$ & $3 \%$ & $3 \%$ & $3 \%$ \\
\hline TOTAL & $\mathbf{1 \%}$ & $\mathbf{4 \%}$ & $\mathbf{3 \%}$ & $\mathbf{1 \%}$ & $\mathbf{1 \%}$ & $\mathbf{1 \%}$ \\
\hline
\end{tabular}
LEGENDA:

Inform. - informática

Admin. - administrativos

N/T - notário/tribunal

Inf. méd. - informação médica

Transp. - Transportes

Os gráficos VI a IX vêm ilustrar os resultados destes quadros. 


\section{Gráfico VI}

\section{COLOCAÇÃO DOS LICENCIADOS POR TIPO DE ACTIVIDADE}

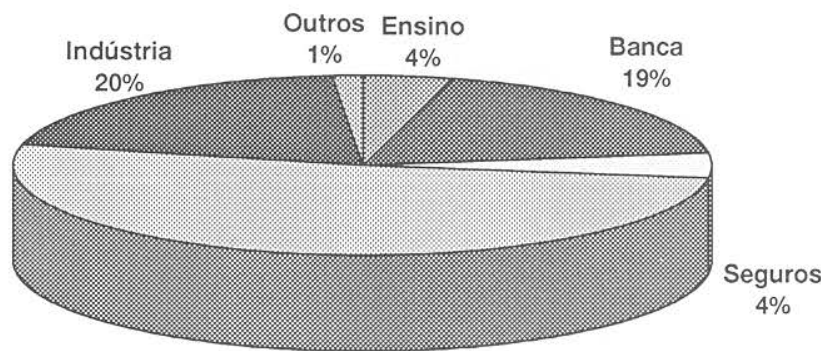

Outros Serviços $52 \%$

\section{Gráfico VII \\ ESPECIFICAÇÃO DOS OUTROS SERVIÇOS}

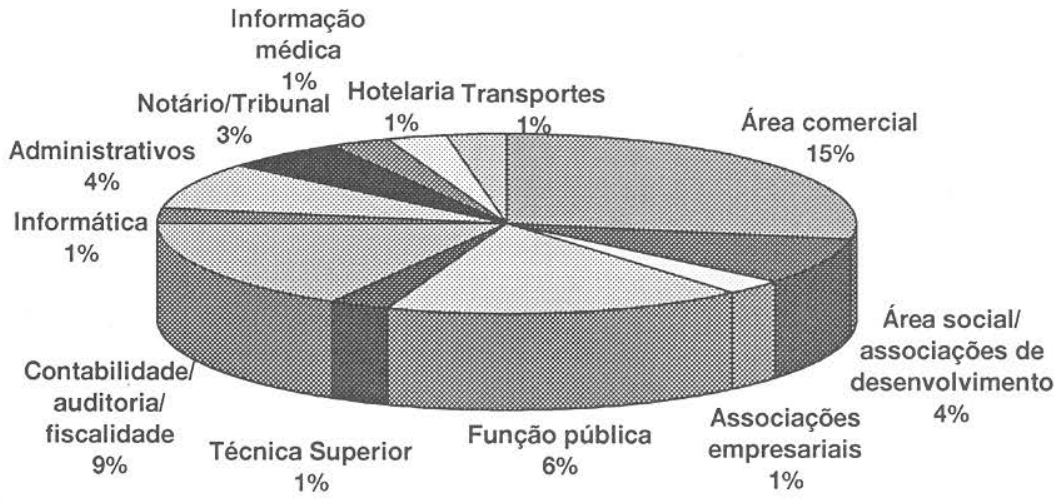




\section{Gráfico VIII \\ COLOCAÇÃO DOS LICENCIADOS POR TIPO DE ACTIVIDADE, POR ANO LECTIVO}

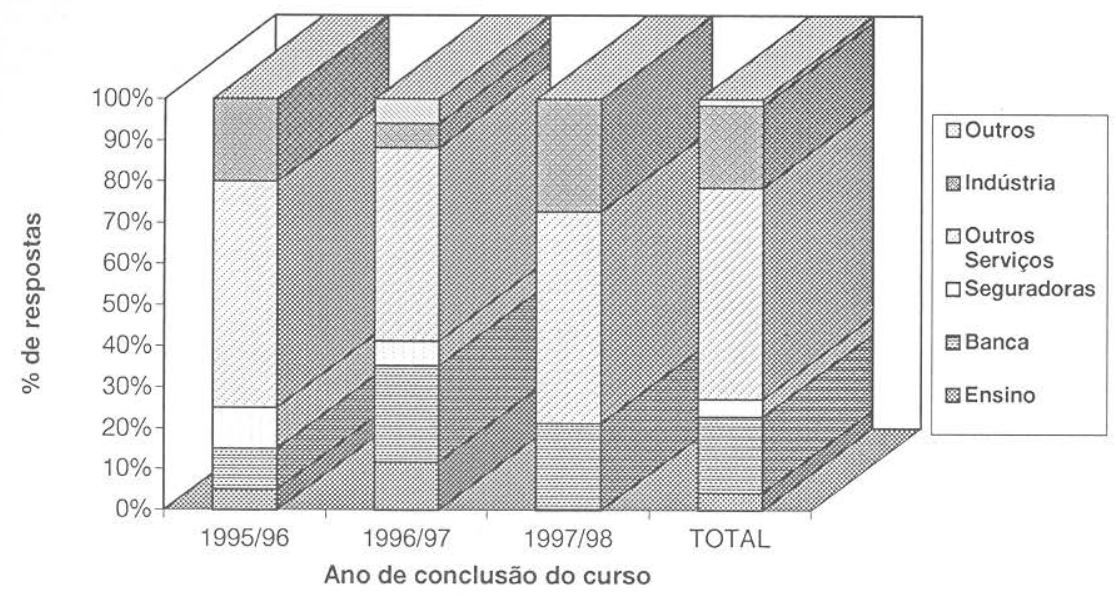

Gráfico IX

ESPECIFICAÇÃO DOS OUTROS SERVIÇOS, POR ANO LECTIVO

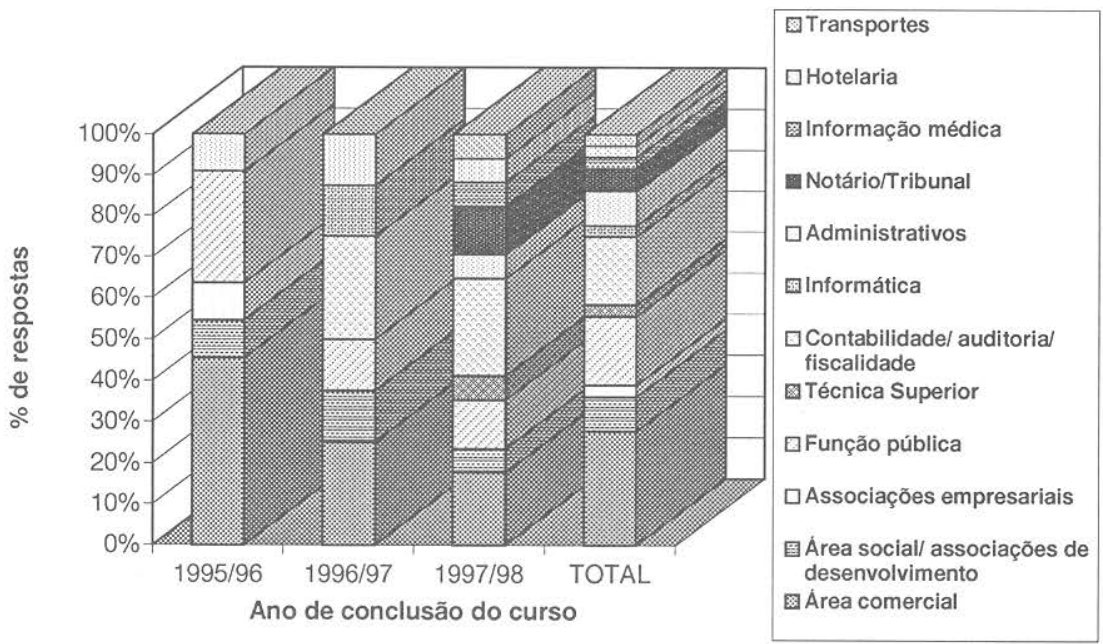


A licenciatura em Gestão e Desenvolvimento Social, ministrada pelo Instituto Universitário de Desenvolvimento e Promoção Social (IUDPS), no Pólo de Viseu, Centro Regional das Beiras da Universidade Católica Portuguesa é, por opção, uma licenciatura de banda larga que integra vários campos das ciências empresariais. Não é, pois, de espantar que as colocações, por tipo de actividade exercida pelos licenciados em $G D S$, sejam tão diversificadas.

O sector terciário é o destino profissional predominante dos licenciados em $G D S$, com $80 \%$ dos licenciados, seguindo-se o sector secundário, com $20 \%$ dos licenciados.

Em termos gerais, a indústria é, como já vimos, o destino de $20 \%$ dos licenciados em GDS. Dentro do sector de serviços, os tipos de actividade predominantemente exercida pelos licenciados em $G D S$ são a banca, destino de $19 \%$ da amostra em análise, 14\% encontram-se na área comercial e 9\% exercem funções nas áreas de contabilidade / auditoria / fiscalidade, estando também 9\% empregados na função pública. Como tipo de actividades exercidas pelos licenciados em $G D S$, com um peso relativo menor nas saídas profissionais, temos o ensino, seguros, área social/associações de desenvolvimento e serviços administrativos (todos com um peso de $4 \%$, entre o total de licenciados empregados), existindo ainda licenciados em $G D S$ colocados em notários / tribunais, em associações empresariais, como técnicos superiores, na área informática, como delegados de informação médica, no sector de hotelaria e em empresas de transportes.

Podem também observar-se as diferenças de colocações, desagregadas por ano lectivo, nos Quadros em análise e no Gráfico VI. Nos três anos lectivos em análise, as considerações mais relevantes quanto às colocações profissionais são o aumento de licenciados colocados na banca, a diminuição de licenciados empregados por seguradoras e na área comercial, existindo também algumas flutuações, para os outros tipos de actividade em análise.

\section{CONSIDERAÇÕES FINAIS}

O presente estudo é uma primeira abordagem à situação profissional dos licenciados em Gestão e Desenvolvimento Social e tem por objectivo fornecer algumas informações que, embora pouco desenvolvidas, possam servir, como primeira análise, de base a estudos mais aprofundados.

Uma primeira consideração tem a ver com a medida da precisão do estudo realizado. Como foram recolhidas informações a partir de uma amostra representativa da população em análise, as estatísticas apresentadas 
são estimativas dos verdadeiros parâmetros da população. Como estimativas, têm associado um determinado erro, que pode ser quantificado.

Para calcular o erro associado às estimativas efectuadas, é necessário ter em conta o conceito de intervalo de confiança para o parâmetro em estudo, neste caso a proporção de licenciados. Este conceito encontra-se em qualquer livro de texto sobre estatística (sugerem-se dois, a título de exemplo: Guimarães e Cabral, 1998; Reis et al., 1996).

O intervalo de confiança para a proporção de licenciados está associado a um nível de confiança, representado em percentagem. Neste estudo iremos utilizar um nível de confiança de $95 \%$, que representa, em média, a proporção de vezes em que o intervalo de confiança contém o parâmetro que se pretende estimar. Os extremos do intervalo designam-se por limites de confiança a $95 \%$ e a semiamplitude do intervalo de confiança corresponde ao erro máximo que, com a confiança especificada, se pode cometer na estimativa do parâmetro proporção de licenciados.

Deste modo, apresentam-se, no Quadro VII, os valores do erro máximo, para um nível de confiança de $95 \%$, associados a algumas das proporções apresentadas no decorrer deste estudo.

\section{Quadro VII \\ ERROS MÁXIMOS ASSOCIADOS AOS RESULTADOS OBTIDOS}

Situação em termos de emprego

\begin{tabular}{|c|c|c|c|c|c|c|}
\hline & Empregados & \multicolumn{3}{|c|}{ Actualmente desempregados } & \multicolumn{2}{|c|}{ Ainda desempregados } \\
\hline Proporção & $87 \%$ & & $7 \%$ & & & $\%$ \\
\hline Erro máximo & $6 \%$ & & $4 \%$ & & 4 & $\%$ \\
\hline \multicolumn{5}{|c|}{ Tempo entre o fim do curso e o $1^{\circ}$ emprego (meses) } & \multicolumn{2}{|c|}{ Rotação no emprego } \\
\hline & $t<0$ & $0<t<1$ & $1<t<6$ & $6<t<12$ & $t>12$ & no $1^{\circ} \mathrm{empr}$. \\
\hline Proporção & $42 \%$ & $21 \%$ & $24 \%$ & $7 \%$ & $6 \%$ & $64 \%$ \\
\hline Erro máximo & $8 \%$ & $7 \%$ & $7 \%$ & $4 \%$ & $4 \%$ & $8 \%$ \\
\hline \multicolumn{7}{|c|}{ Colocação por tipo de actividade } \\
\hline & Ensino & Banca & Seguros & Out.serv. & Indústria & Outros \\
\hline & $4 \%$ & $19 \%$ & $4 \%$ & $51 \%$ & $20 \%$ & $1 \%$ \\
\hline Erro máximo & $3 \%$ & $6 \%$ & $3 \%$ & $8 \%$ & $6 \%$ & $2 \%$ \\
\hline
\end{tabular}

Os erros máximos associados aos resultados apresentados, para este estudo sobre a situação profissional dos licenciados em $G D S$, variam entre $2 \%$ e $8 \%$, para um nível de confiança de $95 \%$. Estes valores, para o erro máximo de cada resultado, traduzem a relação entre as dimensões da amostra e da população, sendo perfeitamente aceitáveis. 
Na presente conjuntura económica e social, pensamos que a taxa de desemprego que ainda afecta o número de licenciados em $G D S$ nos três anos lectivos em estudo se mantém em níveis relativamente baixos. Deve salientar-se que o tempo de espera entre a obtenção do grau de licenciado e a concretização do primeiro emprego é bastante reduzido, estando grande parte dos licenciados empregados ainda antes de terminar a licenciatura.

A colocação dos licenciados por tipo de actividade está de acordo com os objectivos actuais do curso que, como já foi referido, passam por uma formação de banda larga, assegurada por docentes de qualificações académicas adequadas, que prepara os licenciados para as várias saídas profissionais que se lhes oferecem. A indústria, a banca e a área comercial são os tipos de actividades que atraem mais os licenciados em Gestão e Desenvolvimento Social, logo seguidas, entre outras, por actividades na área da contabilidade / auditoria / fiscalidade, na função pública e nos seguros. Há que salientar ainda que apenas $4 \%$ da amostra em análise exerce actividades na área social / associações de desenvolvimento.

Uma conclusão importante que podemos retirar deste estudo é que a licenciatura em Gestão e Desenvolvimento Social, pela análise das saídas profissionais dos licenciados nos anos lectivos em análise: 1995/96, 1996/97 e 1997/98, cada vez se afigura mais como uma licenciatura típica de Gestão, Administração ou Organização de Empresas.

\section{BIBLIOGRAFIA}

GUIMARÃES, R. C.; CABRAL, J. A. Sarsfield (1998), Estatística, Ed. Revista, Ed. McGraw-Hill, Lisboa.

REIS, E.; MELO, P.; ANDRADE, R.; CALAPEZ, T. (1996), Estatística Aplicada, Edições Sílabo, Lisboa.

VICENTE, Paula; REIS, Elisabeth; FERRÃO, Fátima (1996), Sondagens - A Amostragem como Factor Decisivo de Qualidade, Ediçōes Sílabo, Lisboa. 